\title{
Sobre ondas gravitacionais planas não-lineares
}

On non-linear plane gravitational waves

\author{
F.L. Carneiro*10, S.C. Ulhoa ${ }^{1,2,30}$ \\ ${ }^{1}$ Universidade de Brasília, Instituto de Física, Brasília, DF, Brasil. \\ ${ }^{2}$ Universidade de Brasília, Instituto de Física, Centro Internacional de Física, Brasilia, DF, Brasil. \\ ${ }^{3}$ Canadian Quantum Research Center, BC V1T 2L7, Ave Vernon, Canada.
}

Recebido em 30 de março de 2021. Revisado em 19 de maio de 2021. Aceito em 24 de maio de 2021.

\begin{abstract}
As ondas gravitacionais não-lineares são soluções exatas das equações de Einstein que descrevem um campo radiativo propagando com a velocidade da luz. A não-linearidade das equações de Einstein torna difícil o rastreamento de soluções analíticas, sendo necessárias considerações sobre a simetria do problema. Destarte, a obtenção de soluções radiativas, como as ondas gravitacionais de frente plana com raios paralelos - pp-waves (plane-fronted gravitational waves with parallel rays), necessita considerações sobre a simetria e natureza dessas soluções antes das funções restantes serem introduzidas nas equações de Einstein. A obtenção do tensor métrico que descreve as pp-waves envolve avançados conhecimentos matemáticos, tornando a estrutura das deduções não intuitiva. O objetivo deste trabalho é a obtenção do tensor métrico das pp-waves a partir de considerações simples sobre a estrutura da congruência geodésica dessas ondas gravitacionais.
\end{abstract}

Palavras-chave: Ondas gravitacionais não-lineares, Relatividade Geral.

\begin{abstract}
The nonlinear gravitational waves are exact solutions of Einstein's equations that describe a radiative field propagating with the speed of light. The non-linearity of Einstein's equations makes it difficult to track analytical solutions, requiring considerations on the symmetry of the problem. Thus, obtaining radiative solutions, like the pp-waves (plane-fronted gravitational waves with parallel rays), requires considerations about the symmetry and nature of these solutions before the remaining functions are introduced into Einstein's equations. Obtaining a metric tensor that describes the pp-waves involves advanced mathematical knowledge, making the deductions non-intuitive. The main object of this paper is to obtain the pp-waves metric tensor from simple considerations about the geodesic congruence of theses waves space-time.
\end{abstract}

Keywords: Non-linear gravitational waves, General Relativity.

\section{Introdução}

A Física é uma ciência e, como tal, é essencialmente experimental. Apesar do método científico ter passado por embates entre as ideias de indução e dedução, a experimentação prevaleceu como o método mor para se descartar uma determinada hipótese. O objetivo do método científico é chegar ao entendimento mais profundo da natureza, ou seja, estabelecer leis universais. Nesse sentido as hipóteses são descartadas por cada experimento a fim de delimitar determinadas teorias ou expandir o limite de atuação de outras. Por séculos a Física foi essencialmente indutiva e somente um número infinito de corroborações experimentais seria capaz de provar cientificamente uma teoria, ou seja, estabelecendo-a como uma lei universal. Porém, na virada do século XIX para o XX, houve uma mudança de paradigma na Física, a vertente dedutiva assumiu definitivamente um lugar de destaque no meio acadêmico. A assim chamada Física Teórica mostrou sua força quando a Relatividade Geral teve suas previsões corroboradas por alguns experimentos como o

\footnotetext{
*Endereço de correspondência: fernandolessa45@gmail.com
}

desvio da luz. A Teoria da Relatividade Geral, introduzida por Einstein e Hilbert [1, 2], surgiu como resposta a diversos efeitos gravitacionais até então observados, mas que não podiam ser descritos pela teoria Newtoniana da gravitação. Apesar da inovação proposta pela nova teoria, as equações dinâmicas do chamado campo gravitacional, conhecidas como equações de Einstein, possuem um alto grau de não-linearidade na variável fundamental do campo, o tensor métrico. Nesta teoria, o campo gravitacional é uma entidade geométrica, sendo descrito pelo tensor de curvatura, ou tensor de Riemann, que é construído a partir do tensor métrico, possuindo dezesseis componentes independentes em uma variedade (espaço de dimensão e assinatura arbitrárias que se assemelha localmente ao espaço Euclidiano) com quatro dimensões. A equação de campo tensorial resulta em dez equações para o tensor de curvatura. Portanto, a solução completa de um problema necessita considerações físicas adicionais sobre o mesmo, reduzindo o número de variáveis. A primeira solução conhecida fora encontrada por Schwarzschild [3], que considerou a variedade física, comumente chamada espaço-tempo, possuindo simetria espacial esférica e estática, i.e., sem dependência 
temporal. Posteriormente, outras soluções foram encontradas e uma classificação dessas soluções começou a ser feita 4], com algumas possuindo sentido físico claro, enquanto outras abstratas até os presentes dias [5]. As corroborações experimentais da teoria foram feitas nos anos seguintes, passando inclusive pelo Brasil 6 .

Uma classe de soluções das equações de campo despertou o interesse de Einstein e Rosen [7, 8] que, analogamente às ondas eletromagnéticas descobertas no século anterior, representam uma propagação, na velocidade da luz, de uma configuração da geometria do espaço-tempo. Por o efeito gravitacional manifestarse geometricamente na Relatividade Geral (RG), na presença de um campo gravitacional o tensor métrico, que descreve a estrutura geométrica de uma variedade em um sistema de coordenadas específico, sofre uma modificação em relação ao tensor métrico que representa o espaço-tempo plano neste mesmo sistema de coordenadas. Devido à dependência da forma do tensor métrico com o sistema de coordenadas, ele não é adequado para indicar se um espaço-tempo é plano ou não, necessitando o cálculo do tensor de curvatura. A forma do tensor de curvatura depende do sistema de coordenadas, mas a sua nulidade em um ponto implica que o espaço-tempo é plano naquele ponto em particular. Isto não garante que o espaço-tempo seja plano globalmente e nem a não existência de efeitos gravitacionais, uma vez que o fenômeno gravitacional é global e não local. Sendo assim, inicialmente campos gravitacionais distintos podem ser distinguidos entre si pelos escalares de curvatura, que são construídos a partir do tensor de curvatura. Entretanto, os escalares de curvatura das ondas gravitacionais, que são soluções exatas das equações de Einstein, são nulos, não possibilitando a distinção desses espaços-tempo do espaço-tempo plano de Minkowski por meio de tais escalares apenas. Esse problema, além de outros como a exigência, à época, da existência de um sistema de coordenadas que fosse regular em toda a variedade, levou a uma grande incerteza sobre a existência de tais ondas. A incerteza perdurou até o fim dos anos cinquenta, com a negativa à existência sendo defendida pelo próprio Rosen [8]. Em 1957, Bondi contestou a afirmação "tais ondas [gravitacionais polarizadas] não poderiam existir porque a métrica possuiria certas singularidades físicas" [9] de Rosen. Apesar da afirmação, Bondi não apresentou completamente sua discussão, argumentando que as ondas linearmente polarizadas carregam energia, mesmo sem haver um conceito bem estabelecido de energia gravitacional na época. A complementação da discussão veio em outro trabalho realizado com a colaboração de Pirani e Robinson [10], onde as ondas gravitacionais planas foram propriamente definidas como solução exata das equações de Einstein, admitindo a mesma quantidade de simetrias que as ondas eletromagnéticas planas. Apesar das ondas gravitacionais planas serem idealizações, assim como a contraparte eletromagnética, ondas reais podem ser aproximadas por tais ondas quando analisadas a grandes distâncias das fontes reais.

A história da detecção de ondas gravitacionais é quase tão antiga quanto a sua proposição. Por diversas vezes a medição foi anunciada mas depois não confirmada. A primeira vez foi devido a Weber , que utilizou cilindros metálicos como detectores 11. A partir disso diversos eventos ocorreram, inclusive medições indiretas como aquelas advindas da observação de estrelas de nêutrons 12, 13. Diversos projetos foram implementados como o detector Schenberg em São Paulo 14. O mais proeminente dessas iniciativas foi o LIGO que é um projeto desenvolvido para usar interferometria para medir a passagem das ondas gravitacionais. Dessa tentativa resultou a famosa detecção de 2016 [15]. Apesar de existirem cientistas laureados com o prêmio Nobel pelas contribuições no desenvolvimento da detecção de ondas gravitacionais, é preciso ter cautela e esperar medições independentes para a confirmação inequívoca da existência de ondas gravitacionais. O anúncio da detecção por interferometria causou um grande interesse na comunidade científica [16]. Esse interesse levou a um grande número de trabalhos recentes analisando o efeito memória causado por tais ondas [17 19]. O efeito memória é de suma importância na teoria de detecções, uma vez que uma configuração de partículas é permanentemente alterada após a passagem dessas ondas. Estudos posteriores mostraram que o efeito memória é capaz de explicar a propagação dessas ondas por grandes distâncias, sem dissipação, e ainda permitir a detecção a partir da troca de energia localmente com partículas teste [20].

Apesar das ondas gravitacionais exatas serem soluções das equações de Einstein, existe uma categoria de ondas gravitacionais que são soluções aproximadas das equações de Einstein, as linearizadas. Acredita-se que a grandes distâncias as ondas gravitacionais possam perder intensidade e apresentam-se como soluções das equações de Einstein linearizadas, i.e., considerando o fenômeno gravitacional como um campo fraco sobre o espaço-tempo plano. O tensor métrico que representa tais ondas é mais simples de ser obtido e configura exemplos em livros texto padrão [21, 22, assim como em outros trabalhos já presentes na literatura, e.g., veja 23 . Apesar da disponibilidade de textos introdutórios sobre as ondas lineares, as ondas gravitacionais, que de fato são soluções exatas, são pouco exploradas em livros texto. Destarte, o objetivo deste artigo é a construção do tensor métrico para as ondas gravitacionais que são soluções exatas das equações de Einstein, e não soluções aproximadas.

Este artigo está dividido como se segue. Na seção 2 , alguns aspectos básicos (tensores, derivação em variedades e geodésicas), necessários à compreensão da dedução, são revisados. Na seção 3, os conceitos de congruência geodésica nula são descritos, com sua aplicação para a determinação do tensor métrico das pp-waves realizada na seção 4 Por fim, os comentários finais sobre o assunto 
e referências adicionais são apresentados na seção 5 Ao longo deste artigo, o sistema de unidades geometrizado é utilizado, i.e., $G=c=1$.

\section{Teoria geométrica da gravitação}

$\mathrm{Na}$ teoria relativística de Einstein, distintamente da Galileana, o tempo não é absoluto, i.e., observadores distintos podem medir intervalos de tempos distintos entre dois eventos. Desta forma, como as coordenadas espaciais, o tempo é relativo. Para fins de apresentação, pode-se considerar uma variedade fictícia quadridimensional com três eixos formados pelas coordenadas espaciais e o restante pelo tempo [24]. Um ponto nesse espaço fictício é chamado evento e uma curva, nele contida, linha mundo. A consideração dessa variedade na $R G$, chamada espaço-tempo, torna necessário o tratamento dos fenômenos físicos com a geometria de Riemann.

A gravidade é estudada a partir da variação do intervalo, no espaço-tempo, entre dois eventos, ou seja, através do elemento de linha no espaço quadrimensional. Nas coordenadas $x^{\mu}=\left(x^{0}, x^{i}\right)=\left(x^{0}, x^{1}, x^{2}, x^{3}\right)$ o elemento de linha é descrito por

$$
d s^{2}=g_{\mu \nu} d x^{\mu} d x^{\nu},
$$

onde duas letras gregas contraídas indicam um somatório, de acordo com a convenção de Einstein, e as quantidades $g_{\mu \nu}$ são funções das coordenadas $x^{\mu} \mathrm{e}$ suas derivadas. Se o espaço-tempo é plano, i.e., não há efeito gravitacional, ele é chamado espaço-tempo de Minkowski. O espaço plano pode ser descrito em coordenadas Cartesianas $(t, x, y, z)$ por $g_{\mu \nu}=\operatorname{diag}(-1,1,1,1)$, onde o elemento de linha (1) torna-se

$$
d s^{2}=-d t^{2}+d x^{2}+d y^{2}+d z^{2} .
$$

Quando o espaço-tempo plano é descrito por coordenadas Cartesianas, temos $g_{00}=-1, g_{i i}=1$ e $g_{\mu \nu}=0$ para $\mu \neq \nu$, onde $i=1,2,3$. Com isso, um espaço-tempo é dito plano se existe um sistema de coordenadas $x^{\prime \mu}$ em que o elemento de linha (1) pode ser escrito na forma (2); o mesmo é dito curvo se não existe tal sistema de coordenadas.

Tendo em vista a natureza tensorial da teoria relativística de Einstein, faz-se necessário algum conhecimento sobre tensores. Alguns conceitos básicos de tensores são apresentados na subseção 2.1. Nos espaços curvos o conceito usual de derivada deve ser generalizado, sendo feito na subseção 2.2. Devido à trajetória de uma partícula em um campo gravitacional ser resultado da geometria do espaço-tempo na RG, as partículas livres, na ausência de força, possuem como linha mundo uma curva geodésica nessa variedade. Destarte, o conceito de geodésicas é apresentado na subseção 2.3. A presente seção não consiste uma revisão da Teoria da Relatividade Geral, apenas o estabelecimento de alguns conceitos geométricos que serão utilizados ao longo do resto do trabalho. Revisões didáticas da teoria e da geometria Riemanniana podem ser encontradas nas referências [25] 27 .

\subsection{Tensores}

Os tensores são generalizações dos vetores assim como os vetores são dos escalares. Tais entidades geométricas podem ser definidas a partir das regras de transformação de coordenadas. Sejam $x^{\mu}$ e $x^{\prime \mu}$ dois sistemas de coordenadas arbitrários, seus diferenciais estão relacionadas por uma transformação de coordenadas do tipo [24]

$$
d x^{\mu}=\frac{\partial x^{\mu}}{\partial x^{\prime \nu}} d x^{\nu} .
$$

Define-se as quantidades $A^{\mu}$ que se transformam como na equação (3) de quadrivetores contravariantes, ou componentes contravariantes de um quadrivetor. Assim, os componentes contravariantes $A^{\mu}$ de um quadrivetor A são definidos como aqueles que se transformam como

$$
A^{\mu}=\frac{\partial x^{\mu}}{\partial x^{\prime \nu}} A^{\prime \nu}
$$

As quantidades $A_{\mu}$ que se transformam como

$$
A_{\mu}=\frac{\partial x^{\prime \nu}}{\partial x^{\mu}} A_{\nu}^{\prime}
$$

são chamados componentes covariantes $A_{\mu}$ do quadrivetor $\mathbf{A}$. Um quadrivetor $\mathbf{A}$ pode ser escrito tanto em função de seus componentes covariantes quanto contravariantes [21], i.e.,

$$
\mathbf{A}=A^{\mu} \partial_{\mu}=A_{\mu} \partial^{\mu},
$$

onde $\partial_{\mu}$ e $\partial^{\mu}$ formam um sistema recíproco de vetores

$$
\partial^{\mu} \partial_{\nu}=\delta_{\nu}^{\mu}=\left\{\begin{array}{l}
0 \forall \mu \neq \nu \\
1 \forall \mu=\nu
\end{array}\right.
$$

Um escalar pode ser obtido a partir do produto entre os componentes contravariantes e covariantes, sendo invariante sob transformações de coordenadas, i.e.,

$$
A^{\mu} A_{\mu}=\frac{\partial x^{\mu}}{\partial x^{\prime \nu}} \frac{\partial x^{\prime \nu}}{\partial x^{\mu}} A^{\prime \nu} A_{\nu}^{\prime}=A^{\prime \mu} A_{\mu}^{\prime} .
$$

Sendo assim, qualquer entidade geométrica que satisfaz a equação (8) é chamada escalar. Os escalares e vetores são tensores de ordem 0 e 1, respectivamente. Generalizando o acima exposto, pode-se definir tensores $\mathbf{T}$ de ordem mais alta, cujos componentes $T^{\mu \ldots \nu}{ }_{\alpha \ldots \beta}$ se transformam de acordo com

$$
T_{\alpha \ldots \beta}^{\mu \ldots \nu}=\frac{\partial x^{\mu}}{\partial x^{\prime \rho}} \cdots \frac{\partial x^{\nu}}{\partial x^{\prime \lambda}} \frac{\partial x^{\prime \gamma}}{\partial x^{\alpha}} \cdots \frac{\partial x^{\prime \delta}}{\partial x^{\beta}} T^{\prime \rho \ldots \lambda}{ }_{\gamma \ldots \delta} .
$$

Um exemplo de tensor é o tensor unidade $\delta_{\nu}^{\mu}$, descrito pela equação (7). 
A quantidade $d x^{\mu} d x^{\nu}$ na equação (1) se transforma de acordo com

$$
d x^{\mu} d x^{\nu}=\frac{\partial x^{\mu}}{\partial x^{\prime \alpha}} \frac{\partial x^{\nu}}{\partial x^{\prime \beta}} d x^{\prime \alpha} d x^{\prime \beta},
$$

logo, é um tensor. O quadrado do intervalo $d s^{2}$ é um invariante, uma vez que a distância entre dois pontos não depende do sistema de coordenadas adotado. Logo, os componentes $g_{\mu \nu}$ são componentes de um tensor simétrico, chamado tensor métrico. Os mesmos componentes físicos podem ser representadas nas formas covariantes e contravariantes, sendo essas conectadas pelo tensor métrico como

$$
\begin{aligned}
& A^{\mu}=g^{\mu \nu} A_{\nu}, \\
& A_{\mu}=g_{\mu \nu} A^{\nu},
\end{aligned}
$$

com o tensor métrico atuando como operador de levantamento e abaixamento de índices.

Destarte, por os tensores serem entidades geométricas, independem de um sistema de coordenadas. As leis físicas são descrições linguísticas de fenômenos observados. Logo, tais leis não podem depender da descrição escolhida, i.e., do sistema de coordenadas. Essa semelhança torna os tensores a linguagem indicada para as leis da física serem escritas.

\subsection{Derivação Covariante}

As leis da física são independentes do sistema de coordenadas utilizado. Assim, matematicamente, tais leis são melhores descritas por equações que não dependam da escolha de coordenadas. Tais equações são chamadas covariantes. Um exemplo prático é uma curva parametrizada $\gamma$ que pode ser descrita em função de um sistema de coordenadas $x^{\mu}(\lambda)$. A taxa de variação de uma função escalar $f\left(x^{\mu}\right)$ ao longo dessa curva é dada por 28 ]

$$
\frac{d f}{d \lambda}=\frac{\partial f}{\partial x^{\mu}} \frac{d x^{\mu}}{d \lambda} \equiv\left(\partial_{\mu} f\right) u^{\mu} .
$$

A derivada da função $f$ em $\lambda$ é um escalar, sendo descrito pela contração de um vetor (componente) contravariante $\frac{d x^{\mu}}{d \lambda} \equiv u^{\mu}$ e de um vetor (componente) covariante $\frac{\partial f}{\partial x^{\mu}} \equiv$ $\partial_{\mu} f$. O vetor contravariante é tangente à curva $\gamma$ em todo ponto $P$ e, distintamente do caso de uma variedade plana, esse campo vetorial tangente não está contido no mesmo espaço que a curva $\gamma$, como pode ser visto na Figura 1. O conceito de derivada deve ser generalizado para que seja possível a derivação em um espaço-tempo não plano.

A derivada de um vetor contravariante se transforma como

$$
\partial_{\nu}^{\prime} A^{\prime \mu} \equiv \frac{\partial A^{\prime \mu}}{\partial x^{\prime \nu}}=\frac{\partial x^{\prime \mu}}{\partial x^{\alpha}} \frac{\partial x^{\beta}}{\partial x^{\prime \nu}} \frac{\partial A^{\alpha}}{\partial x^{\beta}}+\frac{\partial^{2} x^{\prime \mu}}{\partial x^{\alpha} \partial x^{\beta}} \frac{\partial x^{\beta}}{\partial x^{\nu}} A^{\alpha} .
$$

A partir da equação acima, percebe-se que a quantidade $\partial_{\nu}^{\prime} A^{\prime \mu}$ não é tensorial. Dessa forma, a derivada deve ser

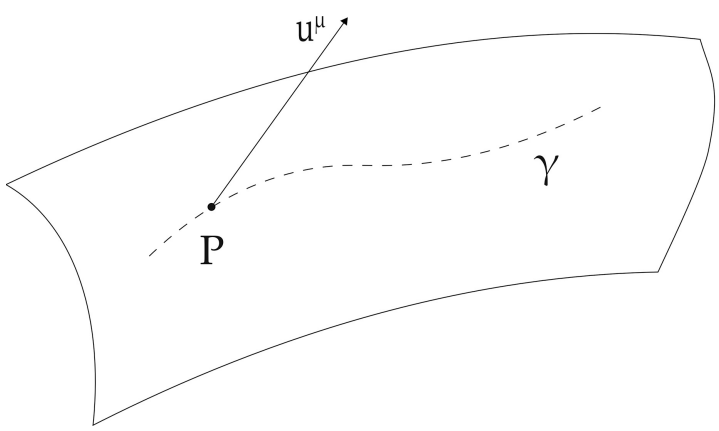

Figura 1: Vetor tangente $u^{\mu}$ a uma curva $\gamma$ no ponto $P$ em uma variedade qualquer.

acrescida de um termo, também não tensorial, que anule o segundo termo da equação (13). Devido à regra de transformação do segundo termo à direita, esse termo adicional deve possuir três índices e satisfazer 28

$$
\Gamma_{\mu \nu}^{\prime \lambda}=\frac{\partial x^{\alpha}}{\partial x^{\prime \mu}} \frac{\partial x^{\beta}}{\partial x^{\prime \nu}} \frac{\partial x^{\prime \lambda}}{\partial x^{\gamma}} \Gamma_{\alpha \beta}^{\gamma}-\frac{\partial^{2} x^{\prime \lambda}}{\partial x^{\alpha} \partial x^{\beta}} \frac{\partial x^{\alpha}}{\partial x^{\prime \mu}} \frac{\partial x^{\beta}}{\partial x^{\prime \nu}} .
$$

Destarte, a derivada de um vetor que se transforma tensorialmente, chamada derivada covariante, é definida para os componentes contravariantes como

$$
\nabla_{\nu} A^{\mu} \equiv \partial_{\nu} A^{\mu}+\Gamma_{\nu \lambda}^{\mu} A^{\lambda}
$$

e para os componentes covariantes como

$$
\nabla_{\nu} A_{\mu} \equiv \partial_{\nu} A_{\mu}-\Gamma_{\mu \nu}^{\lambda} A_{\lambda},
$$

onde quantidade não tensorial $\Gamma_{\mu \nu}^{\lambda}$ chamada conexão afim, deve se transformar sob mudança de coordenadas de acordo com a equação (14). A regra de derivação covariante para vetores pode ser generalizada para tensores de ordem mais alta, i.e.,

$$
\nabla_{\lambda} T^{\mu \nu}=\partial_{\lambda} T^{\mu \nu}+\Gamma_{\gamma \lambda}^{\mu} T^{\gamma \nu}+\Gamma_{\gamma \lambda}^{\nu} T^{\mu \gamma},
$$

onde para cada componente contravariante uma conexão é somada e para cada componente covariante uma conexão é subtraída.

O tensor métrico é um tensor de ordem 2, com sua derivada covariante podendo ser calculada levando-se em conta o fato da derivada covariante de um vetor também ser um vetor. Logo, como $T_{\mu}^{\nu}=\nabla_{\mu} A^{\nu}$, aplicando o tensor métrico obtêm-se

$$
T_{\mu \nu}=g_{\nu \lambda} T_{\mu}^{\lambda} \Rightarrow \nabla_{\mu} A_{\nu}=g_{\nu \lambda} \nabla_{\mu} A^{\lambda} .
$$

Como $A_{\nu}=g_{\nu \lambda} A^{\lambda} \Rightarrow \nabla_{\mu}\left(g_{\nu \lambda} A^{\lambda}\right)=g_{\nu \lambda} \nabla_{\mu} A^{\lambda}$,

$$
\nabla_{\lambda} g_{\mu \nu}=\partial_{\lambda} g_{\mu \nu}+\Gamma_{\mu \lambda}^{\gamma} g_{\gamma \nu}+\Gamma_{\nu \lambda}^{\gamma} g_{\mu \gamma}=0,
$$

i.e., o tensor métrico possui derivada covariante nula. Tal fato permite a conexão ser escrita como

$$
\Gamma_{\mu \nu}^{\lambda}=\bar{\Gamma}_{\mu \nu}^{\lambda}-\frac{1}{2}\left(T_{\mu \nu}^{\lambda}+T_{\nu}^{\lambda}{ }_{\mu}+T_{\nu \mu}^{\lambda}\right),
$$


onde $\bar{\Gamma}_{\mu \nu}^{\lambda}$ é a conexão de Christoffel, que pode ser descrita através do tensor métrico como

$$
\bar{\Gamma}_{\mu \nu}^{\lambda}=\frac{1}{2} g^{\lambda \gamma}\left(\partial_{\mu} g_{\gamma \nu}+\partial_{\nu} g_{\nu \gamma}-\partial_{\gamma} g_{\mu \nu}\right),
$$

e $T_{\mu \nu}^{\lambda}$ é chamado tensor de torção da variedade, definido por

$$
T_{\mu \nu}^{\lambda} \equiv \Gamma_{\mu \nu}^{\lambda}-\Gamma_{\nu \mu}^{\lambda}
$$

A Relatividade Geral é desenvolvida sobre espaçostempo com torção nula, i.e., cuja conexão é simétrica na permutação dos índices inferiores. Teorias construídas em variedades com torção não nula são amplamente estudadas nos presentes dias. Uma delas é o Teleparalelismo Equivalente à Relatividade Geral [29].

\subsection{Geodésicas}

As curvas geodésicas são uma classe de curvas privilegiadas no espaço-tempo. No espaço Euclidiano, uma curva geodésica é facilmente identificada, sendo sempre uma reta conectando dois pontos $P$ e $Q$. Entretanto, em um espaço curvo uma reta pode não estar contida nesse espaço. Logo, faz-se necessária a generalização do conceito de geodésicas, do espaço Euclidiano, para um espaço-tempo curvo. Na RG, as curvas geodésicas são classificadas em três tipos: geodésicas tipo tempo se $d s^{2}<0$; tipo espaço se $d s^{2}>0$; e nulas se $d s^{2}=0$. Para a generalização, duas propriedades importantes devem ser observadas acerca das geodésicas: (i) são curvas que descrevem a menor distância entre dois pontos; (ii) possuem os vetores tangentes a elas todos paralelos entre si [21].

Destarte, pelo critério (i), seja um conjunto de curvas $\gamma$ descritas pelas relações $x^{\mu}(\lambda)$ que passam por dois pontos $P$ e $Q$, onde $\lambda$ é um parâmetro. Uma curva é dita geodésica se ela torna extrema a distância

$$
l=\int_{P}^{Q} L d \lambda=\int_{P}^{Q} \sqrt{ \pm g_{\mu \nu} \dot{x^{\mu}} \dot{x}^{\nu}} d \lambda
$$

onde $\dot{x}^{\mu} \equiv \frac{d x^{\mu}}{d \lambda}$. A distância extrema pode ser obtida pelo princípio variacional. Logo, a equação de EulerLagrange é satisfeita. Sendo assim, pelas equações de Euler-Lagrange, a curva $\gamma$ é geodésica se a relação [28]

$$
\ddot{x}^{\mu}+\bar{\Gamma}_{\alpha \beta}^{\mu} \dot{x}^{\alpha} \dot{x}^{\beta}=\kappa(\lambda) \dot{x}^{\mu}
$$

é satisfeita, onde $\kappa(\lambda) \equiv \frac{d \ln L}{d \lambda}$. Se $\kappa(\lambda)=0$, o parâmetro $\lambda$ pertence a uma classe privilegiada de parâmetros chamados afim [21], e.g., o tempo próprio. Qualquer relação linear com um parâmetro afim, também é um parâmetro afim.

Se $\lambda$ é um parâmetro afim, a equação (23) pode ser escrita como

$$
\frac{D u^{\mu}}{d \lambda}=u^{\nu} \nabla_{\nu} u^{\mu}=0
$$

onde o vetor tangente $u^{\mu}$ à curva $\gamma$ é dado por

$$
u^{\mu}=\frac{d x^{\mu}}{d \lambda}
$$

e definimos o operador $\frac{D}{d \lambda} \equiv u^{\mu} \nabla_{\mu}$. Como $d\left(u^{\mu} u_{\mu}\right) /$ $d \lambda=2\left(\nabla_{\nu} u^{\mu}\right) u_{\nu} u^{\mu}=0$, a variação do vetor $u^{\mu}$ é sempre ortogonal a ele. Sendo assim, uma vez que ele não varia com o parâmetro $\lambda$, ele é dito transportado paralelamente, satisfazendo o critério (ii).

Duas geodésicas inicialmente separadas por um vetor $\xi^{\mu}$ em um espaço-tempo plano mantêm a separação constante ou linear na velocidade. Na presença de um campo gravitacional a alteração da geometria do espaçotempo levará a uma variação não constante no vetor de separação, e.g., duas linhas que seguem paralelas no equador da Terra encontram-se nos polos. Destarte, pode-se computar a curvatura de um espaço-tempo a partir do chamado desvio da geodésica, i.e., a taxa variável no qual uma geodésica afasta-se de outra geodésica padrão. Assim, um espaço-tempo é curvo sempre que

$$
\begin{aligned}
\frac{D^{2} \xi^{\mu}}{d \lambda^{2}} & =\frac{D}{d \lambda}\left(u^{\nu} \nabla_{\nu} \xi^{\mu}\right) \\
& =\left(\nabla_{\alpha} \nabla_{\beta} \xi^{\mu}\right) u^{\alpha} u^{\beta}-\left(\nabla_{\beta} \nabla_{\alpha} \xi^{\mu}\right) u^{\alpha} u^{\beta},
\end{aligned}
$$

é não nulo, onde $\lambda$ é um parâmetro afim. O fenômeno gravitacional pode ser determinado a partir da não nulidade da equação (26). Duas derivadas covariantes não comutam entre si, como ocorre com as derivadas comuns. No caso do espaço-tempo ser plano, as derivadas covariantes tornam-se derivadas comuns, comutando e tornando a equação acima nula. Logo, a curvatura do espaço-tempo relaciona-se à não comutatividade das derivadas parciais, permitindo a definição do tensor de curvatura

$$
R_{\nu \alpha \beta}^{\mu} \equiv\left(\nabla_{\alpha} \nabla_{\beta}-\nabla_{\beta} \nabla_{\alpha}\right),
$$

que possui dezesseis componentes independentes. O tensor de curvatura pode ser escrito a partir das conexões de Christoffel como

$R_{\nu \alpha \beta}^{\mu}=\partial_{\alpha} \bar{\Gamma}_{\nu \beta}^{\mu}-\partial_{\beta} \bar{\Gamma}_{\nu \alpha}^{\mu}+\bar{\Gamma}_{\rho \alpha}^{\mu} \bar{\Gamma}_{\nu \beta}^{\rho}-\bar{\Gamma}_{\rho \beta}^{\mu} \bar{\Gamma}_{\nu \alpha}^{\rho}$.

Por o tensor de curvatura nulo em todo o espaçotempo implicar a ausência de campo gravitacional, é possível determinar se um espaço-tempo é curvo univocamente sem a necessidade da tentativa de infinitas transformações de coordenadas que levam o elemento de (1) ao elemento de linha (2). Contraindo o primeiro e o terceiro índices do tensor de curvatura, define-se o tensor simétrico de Ricci $R_{\mu \nu}$, i.e.

$$
R_{\mu \nu} \equiv R_{\mu \rho \nu}^{\rho}
$$

Contraindo os índices do tensor de Ricci obtêm-se o escalar de curvatura

$$
R \equiv g^{\mu \nu} R_{\mu \nu}
$$


A partir dos tensores 29 e é possível definir o tensor de Einstein $G_{\mu \nu}$

$$
G_{\mu \nu} \equiv R_{\mu \nu}-\frac{1}{2} g_{\mu \nu} R
$$

cuja derivada covariante é nula, i.e., $\nabla_{\mu} G^{\mu \nu}=0$. Como a derivada covariante do tensor energia-momento $T_{\mu \nu}$ dos campos de matéria-radiação, que agem como fonte dos campos gravitacionais, também é nula [21, têm-se

$$
G_{\mu \nu}=\kappa T_{\mu \nu}
$$

onde $\kappa=8 \pi\left(8 \pi G c^{-4}\right.$ no SI $)$ é uma constante. As dez equações 32 são chamadas equações de Einstein e descrevem a geometria do espaço-tempo para um dado tensor energia-momento de uma fonte.

\section{Congruências geodésicas nulas}

As equações de Einstein (32) impõem algumas dificuldades de cálculo por serem não-lineares no tensor métrico, além de serem dez equações disponíveis para a determinação de dezesseis componentes do tensor de curvatura. Tal estrutura das equações de campo torna o rastreamento de soluções analíticas uma tarefa impossível sem o uso de considerações adicionais sobre as simetrias do campo gravitacional. O conceito de geodésicas, apresentado na subseção 2.3 . fornece uma maneira de contornar o problema.

Uma congruência geodésica é um conjunto de curvas geodésicas, cujo campo tangente é descrito por $u^{\mu}$, que não se interceptam [28. Um exemplo de congruência é apresentado na Figura 2 A evolução da congruência é medida considerando-se o desvio $\xi^{\mu}$ das geodésicas (linhas pontilhadas) a partir de uma geodésica de referência (linha contínua). A congruência pode ser tipo tempo se formada por geodésicas temporais; nulas se formada por geodésicas nulas; e tipo espaço se formadas por geodésicas espaciais. Como as pp-waves descrevem uma configuração que se propaga com a velocidade

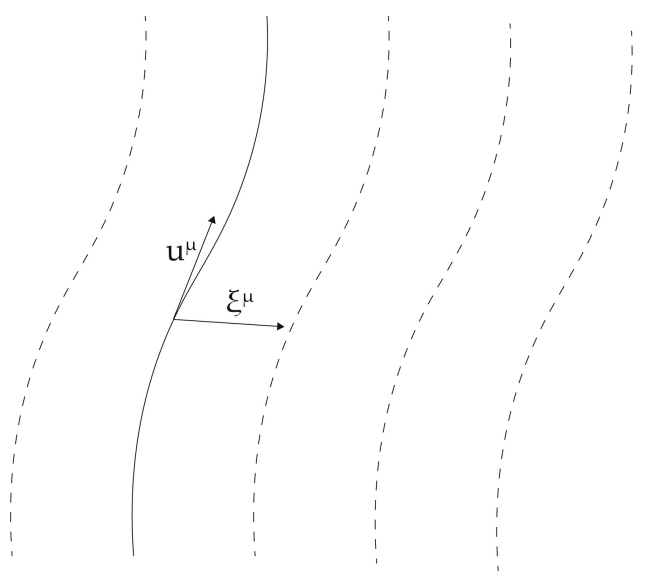

Figura 2: Congruência geodésica com campo tangente $u^{\mu}$ e vetor de separação $\xi^{\mu}$. da luz, o espaço-tempo das pp-waves consiste em uma congruência de geodésicas nulas. Destarte, o foco desse artigo dar-se-á em congruências nulas.

Um observador co-movente, cuja quadrivelocidade possui apenas componente temporal em um sistema de coordenadas, i.e., $u^{\mu}=(-1,0,0,0)$, obedece à relação

$$
u^{\mu} u_{\mu}=-1 \text {. }
$$

A equação acima é uma equação tensorial, sendo válida em qualquer sistema de coordenadas, carregado pelo observador que segue a geodésica ou não. Por o vetor $u^{\mu}$ ser identificado com a direção de evolução do parâmetro afim, e.g., o tempo, convém separar o tensor métrico em duas partes: uma paralela a $u^{\mu}$ outra perpendicular. Para tal, há a necessidade de introduzir-se um vetor nulo $k^{\mu}$, i.e.,

$$
k^{\mu} k_{\mu}=0
$$

chamado principal. Para a separação ser realizada, é necessário a introdução de outro sistema de coordenadas, no qual define-se uma coordenada nula principal $u=\frac{t-z}{\sqrt{2}}$ (orientada para o futuro) e uma coordenada nula auxiliar $v=\frac{t+z}{\sqrt{2}}$ observando o que ocorre em uma geodésica nula sobre um ponto $\mathcal{P}$. As coordenadas nulas são ortogonais entre si e às coordenadas espaciais $x, y$ restantes. A construção pode ser visualizada observando o que ocorre em uma geodésica nula sobre o ponto $\mathcal{P}$ na Figura 3 No novo sistema de coordenadas, o elemento de linha do espaço-tempo de Minkowski fica escrito como

$$
d S^{2}=-d u d v+d x^{2}+d y^{2} .
$$

Se $k^{\mu}$ é tangente às curvas $u=$ constante, o elemento de linha do espaço transverso se torna $d s^{2}=d x^{2}+d y^{2}$. Neste caso, introduz-se outro vetor nulo $N^{\mu}$, chamado de vetor nulo auxiliar, paralelo à $k^{\mu}$, i.e.,

$$
N^{\mu} N_{\mu}=0
$$

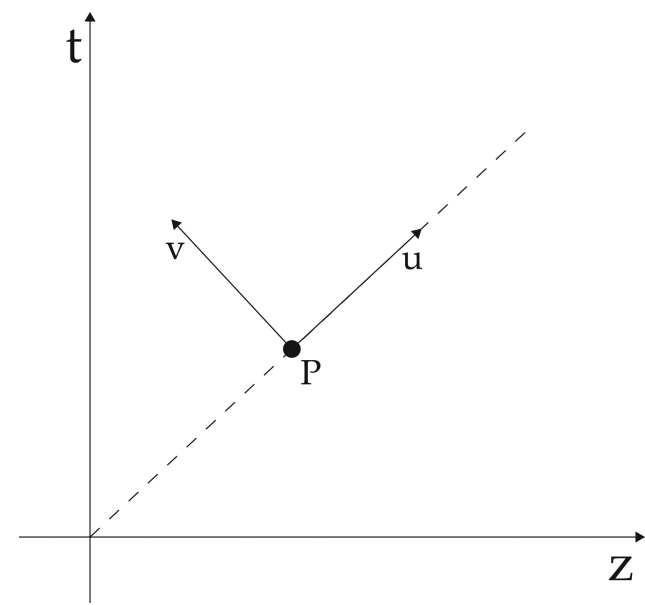

Figura 3: Coordenadas nulas $u$ e $v$ em um diagrama $t z$. 
e

$$
N^{\mu} k_{\mu}=-1,
$$

onde o sinal negativo indica que ambos apontam para o futuro. A partir dos vetores nulos $k^{\mu}$ e $N^{\mu}$ é possível a construção de um tensor métrico que representa o espaço transverso às direções nulas. Denotando esse tensor métrico por $h_{\mu \nu}$, ele pode ser definido como 28

$$
h_{\mu \nu} \equiv g_{\mu \nu}+k_{\mu} N_{\nu}+N_{\mu} k_{\nu},
$$

de forma a deixar o elemento de linha no espaço transverso como $d S^{2}=h_{\mu \nu} d x^{\mu} d x^{\nu}=d x^{2}+d y^{2}$.

A métrica transversa dada pela equação 38 permite a descrição das congruências nulas no espaço-tempo curvo. Ela possui algumas propriedades, sendo elas que $h_{\mu \nu}$

1. é ortogonal à $k^{\mu}$, i.e., $h_{\mu \nu} k^{\mu}=0$;

2. é ortogonal à $N^{\mu}$, i.e., $h_{\mu \nu} N^{\mu}=0$;

3. possui traço 2 , i.e., $h_{\mu}^{\mu}=2$;

4. projetado nele mesmo gera ele mesmo, i.e., $h_{\rho}^{\mu} h_{\nu}^{\rho}=h_{\nu}^{\mu}$.

Decerto a escolha para a métrica transversa não é única, uma vez que para uma mesma direção nula principal $k^{\mu}$ existem infinitas direções nulas auxiliares que satisfazem as equações (36 37] 28.

Na presença de um campo gravitacional, as geodésicas pontilhadas na Figura 2 afastar-se-ão da geodésica de referência (contínua). A taxa de afastamento das geodésicas da geodésica de referência pode ser medida pela variação do vetor separação

$$
B_{\mu \nu} \equiv \nabla_{\nu} k_{\mu}
$$

onde o tensor $B_{\mu \nu}$ representa a falha de $\xi^{\mu}$ ser transportado ao longo da congruência, i.e., $\left(\nabla_{\nu} \xi^{\mu}\right) k^{\nu}=B^{\mu}{ }_{\nu} \xi^{\nu}$. Apesar de $B_{\mu \nu}$ ser ortogonal à $k^{\mu}$, ele não é à $N^{\mu}$. Como a separação das curvas nulas ocorre no espaço transverso, é necessária a remoção dos componentes paralelos ao vetor nulo auxiliar $N^{\mu}$. Para tal, o vetor desvio pode ser projetado no espaço transverso, i.e.,

$$
\tilde{\xi}^{\mu} \equiv h_{\nu}^{\mu} \xi^{\nu}=\xi^{\mu}+\left(N^{\nu} \xi_{\nu}\right) k^{\mu} .
$$

A evolução do vetor desvio transverso $\tilde{\xi}^{\mu}$ ao longo da congruência é portanto

$$
\left(\nabla_{\nu} \tilde{\xi}^{\mu}\right) k^{\nu}=h_{\rho}^{\mu} B_{\nu}^{\rho} \xi^{\nu}+\left(\nabla_{\nu} N_{\rho}\right) \xi^{\rho} k^{\mu} k^{\nu} .
$$

A expressão acima possui componente na direção $k^{\mu}$. Como o interesse é uma velocidade transversal relativa, novamente a expressão acima é projetada no espaço transverso, obtendo-se

$$
\begin{aligned}
h_{\rho}^{\mu}\left(\nabla_{\nu} \tilde{\xi}^{\rho}\right) k^{\nu} & =h^{\mu}{ }_{\rho} h^{\gamma}{ }_{\nu} B^{\rho}{ }_{\gamma} \tilde{\xi}^{\nu} \\
& =\tilde{B}^{\mu}{ }_{\nu} \tilde{\xi}^{\nu}
\end{aligned}
$$

onde defini-se o tensor $B_{\mu \nu}$ projetado $\tilde{B}^{\mu}{ }_{\nu} \equiv$ $h^{\mu}{ }_{\rho} h^{\gamma}{ }_{\nu} B^{\rho}{ }_{\gamma}$. O tensor $\tilde{B}^{\mu \nu}$ não possui nenhuma simetria, tornando sua interpretação física mais intricada.
Isto ocorre por o tensor $\tilde{B}^{\mu \nu}$ ser uma combinação de três partes com propriedades simétricas distintas. Destarte, o tensor $\tilde{B}^{\mu \nu}$ pode ser dividido em uma parte diagonal $\delta_{\mu \nu} \Theta$, uma simétrica $\sigma_{\mu \nu}$ e uma anti-simétrica $\omega_{\mu \nu}$. Assim,

$$
\tilde{B}_{\mu \nu}=\frac{1}{2} \Theta h_{\mu \nu}+\sigma_{\mu \nu}+\omega_{\mu \nu}
$$

onde

$$
\begin{aligned}
& \Theta=\tilde{B}_{\mu}^{\mu}=\nabla_{\mu} k^{\mu}, \\
& \sigma_{\mu \nu}=\tilde{B}_{(\mu \nu)}-\frac{1}{2} \Theta h_{\mu \nu}, \\
& \omega_{\mu \nu}=\tilde{B}_{[\mu \nu]},
\end{aligned}
$$

onde os os parênteses indicam simetrização $A_{(\mu \nu)}=\frac{1}{2}$ $\left(A_{\mu \nu}+A_{\nu \mu}\right)$ e os colchetes anti-simetrização $A_{[\mu \nu]} \stackrel{2}{=}$ $\frac{1}{2}\left(A_{\mu \nu}-A_{\nu \mu}\right)$.

A não nulidade da parte diagonal do tensor $\tilde{B}^{\mu \nu}$, i.e., $\delta_{\mu \nu} \Theta$, produz um afastamento de um conjunto de geodésicas inicialmente paralelas, sendo conhecido como tensor de expansão de uma congruência. Se a congruência converge, e.g., no problema das linhas na superfície da Terra, tem-se $\Theta<0$; se diverge, $\Theta>0$. $\mathrm{O}$ seu efeito em uma congruência geodésica pode ser visualizado na Figura 4. A expansão $\Theta$ não depende da escolha do vetor nulo auxiliar $N^{\mu}$ 28. O tensor $\sigma_{\mu \nu}$ é conhecido como tensor de distensão, com seu efeito podendo ser visualizado na Figura 5 em um conjunto de geodésicas perpendiculares ao plano $\xi^{x}, \xi^{y}$. O tensor de rotação $\omega_{\mu \nu}$ rotaciona um conjunto de curvas geodésicas que formam uma circunferência no mesmo plano, conforme pode ser visto na Figura 6 .

A evolução da congruência pode ser medida computando-se a variação da expansão $\Theta$ com o

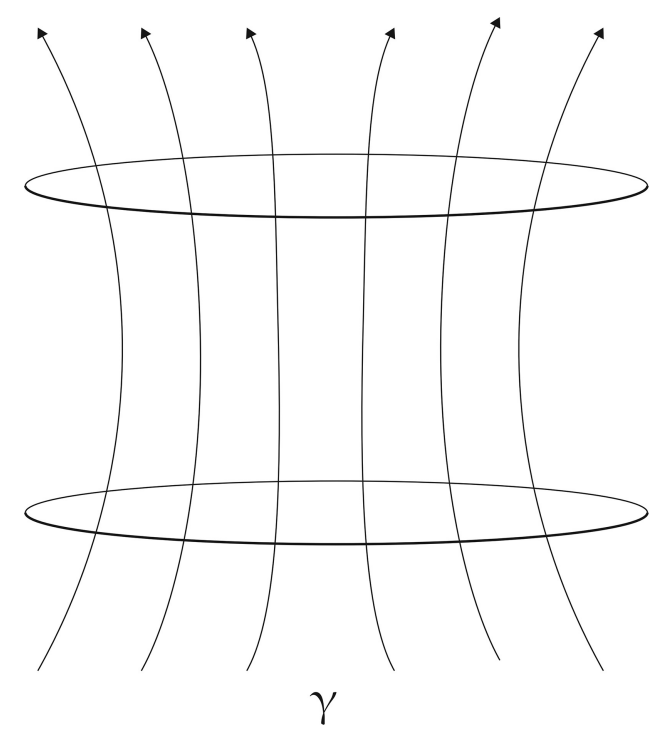

Figura 4: Expansão de uma congruência geodésica. 


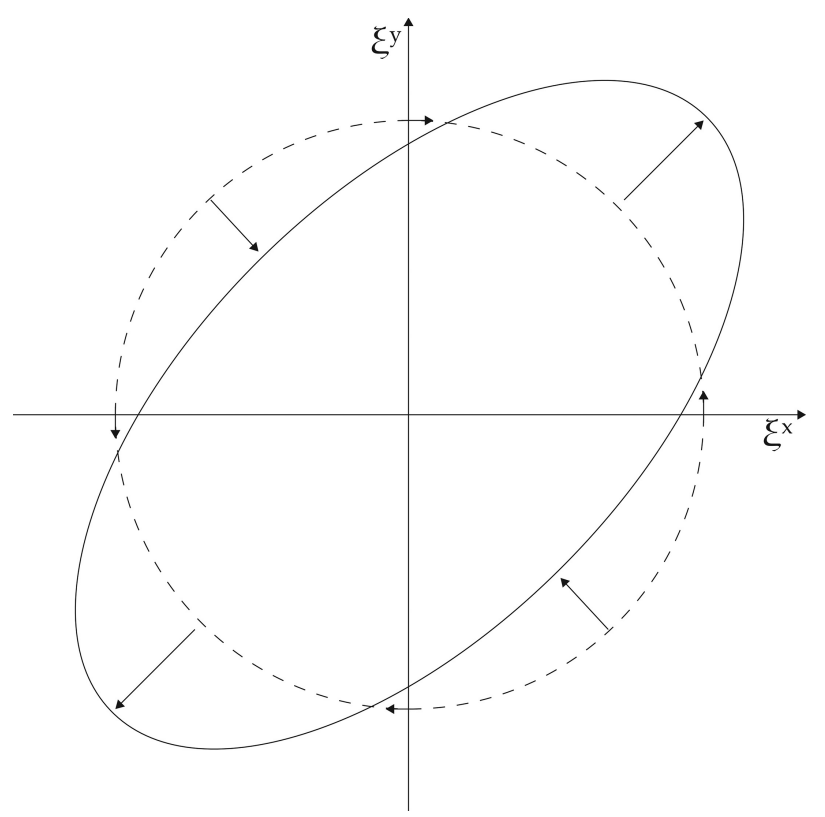

Figura 5: Distensão de uma circunferência de geodésicas (pontilhada) em uma visão transversal.

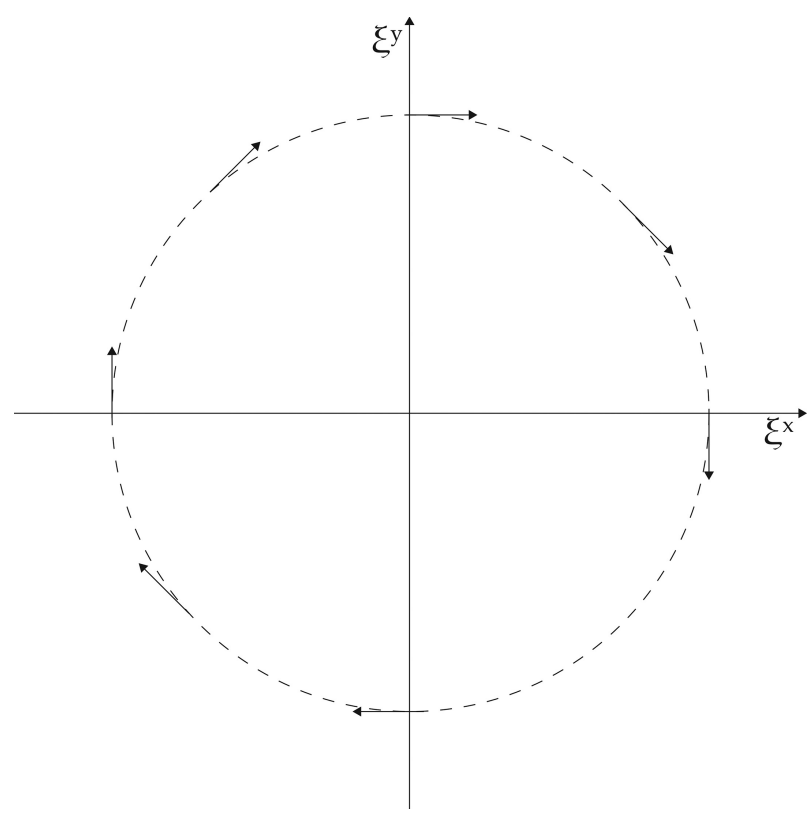

Figura 6: Rotação de uma circunferência de geodésicas (pontilhada) em uma visão transversal.

parâmetro afim $\lambda$, i.e.,

$$
\frac{d \Theta}{d \lambda}=-B^{\mu \nu} B_{\mu \nu}-R_{\mu \nu} k^{\mu} k^{\nu} .
$$

É possível mostrar que $B^{\mu \nu} B_{\mu \nu}=\tilde{B}^{\mu \nu} \tilde{B}_{\mu \nu}$ de maneira a obter-se

$$
\frac{D B_{\mu}^{\mu}}{d \tau}=\frac{D \Theta}{d \tau}=-\frac{1}{2} \Theta^{2}-\sigma^{\mu \nu} \sigma_{\mu \nu}+\omega^{\mu \nu} \omega_{\mu \nu}-R_{\mu \nu} k^{\mu} k^{\nu} .
$$

A equação acima é conhecida como equação de Raychaudhuri 28]. No caso de espaços-tempo cuja congruência geodésica não possui rotação, a relação

$$
\frac{D B_{\mu}^{\mu}}{d \tau} \leq 0
$$

é satisfeita desde que a chamada condição de energia nula $R_{\mu \nu} k^{\mu} k^{\nu}>0$ seja satisfeita. Como para as fontes conhecidas a condição de energia nula é satisfeita, a equação (49), conhecida como Teorema do Foco, pode ser interpretada como uma relação que os campos gravitacionais reais devem satisfazer. Logo, espera-se que nos espaços-tempos reais a congruência geodésica seja convergente, i.e., os campos gravitacionais são atrativos. É interessante notar que nas proximidades de uma singularidade o campo gravitacional pode se tornar repulsivo 30. Isso pode advogar contra a existência física das singularidades nuas.

\section{A métrica das ondas gravitacionais planas}

Usualmente as ondas gravitacionais são introduzidas como uma aproximação de campo fraco das equações de Einstein. Tal aproximação é realizada pela seguinte escolha da métrica

$$
g_{\mu \nu}=\eta_{\mu \nu}+h_{\mu \nu},
$$

onde $\eta_{\mu \nu}$ é a métrica do espaço plano e $h_{\mu \nu}$ é a sua perturbação de primeira ordem. Com isso a equação de campo se reduz a

$$
\nabla_{\alpha} \nabla^{\alpha} \psi_{\mu \nu}=-2 k T_{\mu \nu}
$$

onde $\psi_{\mu \nu}=h_{\mu \nu}-\frac{1}{2} \eta_{\mu \nu} h$ e $h=h_{\nu}^{\nu}$. No vácuo essa equação se reduz a uma equação de onda, ja que $\nabla_{\alpha} \nabla^{\alpha}$ é o operador dalambertiano, daí a motivação para que tais soluções sejam conhecidas como ondas gravitacionais. Nesse artigo queremos mostrar outra abordagem para a introdução de ondas gravitacionais. Elas serão introduzidas como soluções exatas das equações de Einstein em analogia às ondas eletromagnéticas planas. As equações dinâmicas do campo eletromagnético, conhecidas como equações de Maxwell, admitem a existência de soluções na ausência de fonte. Tais soluções representam campos eletromagnéticos que se propagam com a velocidade da luz, chamadas ondas eletromagnéticas planas. Tais ondas consistem uma idealização, uma vez que não se espera sua existência sem uma fonte. Entretanto, soluções reais a grandes distâncias podem ser aproximadas por ondas eletromagnéticas planas. As ondas podem ser descritas por curvas, que nunca se cruzam, paralelas à direção de propagação. Destarte, a congruência dessas não possui expansão, distorção e rotação.

As equações de Einstein também admitem soluções dessa forma, as chamadas pp-waves (plane-fronted gravitational waves with parallel rays), i.e., ondas gravitacionais de frente plana com raios paralelos. Conforme 
visto na subseção 2.3 a não-linearidade das equações de Einstein impossibilita o rastreamento de soluções simplesmente colocando o vácuo como fonte, i.e., $T_{\mu \nu}=$ 0. Para o tensor métrico ser simplificado, algumas paridades com as ondas eletromagnéticas planas devem ser feitas.

As pp-waves se propagam na velocidade da luz, logo, como visto na seção 3 a congruência geodésica desse espaço-tempo deve ser construída a partir de duas coordenadas nulas $(u, v)$ e duas espaciais $(x, y)$. Uma congruência nula possui uma direção nula principal $k^{\mu}$ e uma nula auxiliar $N^{\mu}$. Se a coordenada nula $u=x^{0}$ for identificada como sendo a coordenada que varia na direção nula principal da congruência, a direção $k^{\mu}$ pode ser escrita como sendo o gradiente dessa coordenada [22], i.e.,

$$
k_{\mu}=\partial_{\mu} u=(1,0,0,0) .
$$

Como o espaço-tempo deve possuir raios nulos paralelos normais às superfícies espaciais da frente de onda plana, esses raios paralelos, associados à direção nula principal $k_{\mu}$ da congruência, devem ser covariantemente constantes 31. Logo,

$$
\nabla_{\nu} k_{\mu}=0
$$

Sendo assim, pela equação $(39)$, o tensor $B^{\mu}{ }_{\nu}$ é nulo. A partir da nulidade do tensor $B^{\mu}{ }_{\nu}$, conclui-se que um espaço-tempo que satisfaz a condição (51) não possui expansão $\Theta$, rotação $\omega_{\mu \nu}$ e distensão $\sigma_{\mu \nu}$. Dessa forma, pela equação de Raychaudhuri (48), o tensor de Ricci

$$
R_{\mu \nu}=0 .
$$

As condições 50:51:52 representam as propriedades requeridas de analogia com as ondas eletromagnéticas planas. A solução buscada para as pp-waves deve satisfazer tais condições.

Um mesmo espaço-tempo pode ser descrito por elementos de linha distintos. O elemento de linha das pp-waves é mais facilmente construído nas chamadas coordenadas de Brinkmann [32, onde $x^{0}=u, x^{1}=$ $x, x^{2}=y, x^{3}=v$. Como as ondas possuem uma frente bidimensional plana, o espaço-tempo nas hipersuperfícies $u=$ constante coincide com o espaço-tempo plano bidimensional. Dessa forma, o elemento de linha das pp-waves deve ser um caso particular do elemento de linha

$d s^{2}=g_{00} d u^{2}+2 g_{03} d u d v+2 g_{01} d u d x+2 g_{02} d u d y+d x^{2}+d y^{2}$,

onde, a princípio, as funções do tensor métrico são funções de $u, x, y, v$. Como $u=c t e \Rightarrow d u=0$, a equação (53) torna-se, neste caso,

$$
\left.d S^{2}\right|_{u=c t e}=d x^{2}+d y^{2},
$$

i.e., o elemento de linha do espaço-tempo bidimensional plano. Destarte, as hiper-superfícies $u=c t e$ denotam as frente planas da onda. A direção nula auxiliar $N_{\mu}$ segue a direção do eixo gerado pelo gradiente da coordenada nula auxiliar $v$, logo,

$$
N_{\mu}=\partial_{\mu} v=(0,0,0,1) .
$$

Dessa forma, substituindo 50 e 55 em (37) obtêm-se

$$
k_{\mu} N^{\mu}=-1 \Rightarrow g^{\mu \nu} k_{\mu} N_{\mu}=-1 \Rightarrow g^{03}=-1 .
$$

Invertendo o tensor métrico associado ao elemento de linha 53 , obtêm-se $g_{03}=1 / g^{03}$. Destarte,

$d s^{2}=g_{00} d u^{2}-2 d u d v+2 g_{01} d u d x+2 g_{02} d u d y+d x^{2}+d y^{2}$.

Um vetor $\xi^{\mu}$ que indica uma direção de simetria no espaço-tempo satisfaz a equação

$$
\nabla_{\mu} \xi_{\nu}+\nabla_{\nu} \xi_{\mu}=0
$$

chamada equação de Killing. Como o espaço-tempo deve ser o mesmo em todas as hiper-superfícies $u=$ contante, ele deve possuir um vetor de Killing na direção $k_{\mu}$. Sendo assim, o vetor $k^{\mu}=g^{\mu \nu} k_{\nu}=(0,0,0,1)$ deve ser um vetor de Killing. Construindo as derivadas covariantes para o elemento de linha (57) e substituindo o vetor $k_{\mu}$ nas equações de Killing (58), obtêm-se que o vetor $k^{\mu}$ é um vetor de Killing se e somente se as funções da métrica não forem funções da coordenada $v$. O elemento de linha pode novamente ser reescrito, dessa vez definindo $g_{00} \equiv$ $H(u, x, y), g_{01} \equiv a_{1}(u, x, y)$ e $g_{02} \equiv a_{2}(u, x, y)$. Assim,

$$
\begin{aligned}
d s^{2}= & H(u, x, y) d u^{2}+a_{1}(u, x, y) d u d x \\
& +a_{2}(u, x, y) d u d y+d x^{2}+d y^{2}-2 d u d v
\end{aligned}
$$

O elemento da linha acima é o elemento de linha das ppwaves. Localmente, uma mudança de coordenadas pode ser realizada e o elemento de linha reescrito em outro sistema de coordenadas, onde $g_{01}=g_{02}=0$ [33]. Assim,

$$
d s^{2}=H(U, X, Y) d U^{2}+d X^{2}+d Y^{2}-2 d U d V .
$$

Nas coordenadas $(U, X, Y, V)$ o tensor métrico fica então escrito como

$$
g_{\mu \nu}=\left(\begin{array}{cccc}
H & 0 & 0 & -1 \\
0 & 1 & 0 & 0 \\
0 & 0 & 1 & 0 \\
-1 & 0 & 0 & 0
\end{array}\right) .
$$

Para a completa resolução do problema, resta apenas a determinação da função $H(U, X, Y)$. Entretanto, o problema está simplificado o suficiente, permitindo a introdução do tensor métrico (61) nas equações de Einstein. Os termos da conexão de Christoffel podem ser calculados diretamente a partir da equação 20; e, a partir da conexão, os componentes do tensor de curvatura $(28)$ e do tensor de Ricci $(29)$. Substituindo o tensor de Ricci nas equações de Einstein (32) com $T_{\mu \nu}=0$, ou diretamente na equação 52 , obtêm-se [31]

$$
\partial_{X}^{2} H+\partial_{Y}^{2} H=0
$$


Várias soluções particulares, polarizações distintas, podem ser construídas a partir da equação 62. As equações de Einstein não fornecem restrições sobre a dependência na variável $U$ da função $H$. Essa liberdade é justamente a escolha do formato do pulso da onda a ser considerada.

A função $H(U, X, Y)$ determina a característica e a existência das ondas gravitacionais. Em sua ausência, o espaço-tempo de Minkowski é recuperado, logo, para $H(u, x, y)=0$,

$$
d s^{2}=d X^{2}+d X^{2}-d U d V=-d t^{2}+d x^{2}+d y^{2}+d z^{2} .
$$

Sendo assim,

$$
\begin{aligned}
-2 d U d V & =(d t-d z)(d t+d z) \Rightarrow d U d V \\
& =\frac{(d t-d z)}{\sqrt{2}} \frac{(d t+d z)}{\sqrt{2}} .
\end{aligned}
$$

Como as frentes de onda são hiper-superfícies nulas, a frente de onda estará sempre na coordenada $U=0$. Logo, as coordenadas $U, V$ podem ser identificadas, no limite do espaço-tempo plano, com as coordenadas Cartesianas a partir das relações

$$
U=\frac{t-z}{\sqrt{2}}
$$

e

$$
V=\frac{t+z}{\sqrt{2}}
$$

A coordenada $z$ nas equações acima representa a direção de propagação da onda gravitacional sempre que a direção nula principal da congruência for $k^{\mu}$, i.e., o gradiente da coordenada $U$.

\section{Conclusões}

Após a introdução da Teoria da Relatividade Geral, grande parte dos livros texto apresentam a solução de Schwarzschild como exemplo padrão. Entretanto, as pp-waves são uma classe de soluções simples das equações de Einstein, possuindo clara analogia com o eletromagnetismo. As suposições sobre a simetria do problema não são difíceis de serem visualizadas e os procedimentos de simplificação da solução, para o posterior uso nas equações de Einstein, possuem o mesmo nível de complexidade que a solução de Schwarzschild. Neste artigo o elemento de linha das ondas gravitacionais planas (60) fora apresentado a partir de uma extensão do conceito de congruências geodésicas, utilizando propriedades fundamentais das ondas eletromagnéticas como conceito subsunçor.

As ondas gravitacionais linearizadas são amplamente apresentadas em livros texto, mas não fornecem um conhecimento introdutório sobre soluções exatas das equações de Einstein. O estudo das ondas gravitacionais planas auxilia tanto a exemplificação de algumas soluções das equações de campo como fornece conhecimento sobre as mesmas. Conhecimento esse amplamente necessário nos presentes dias, dado o grande número de pesquisas teóricas e observações experimentais apresentados na literatura.

Dentro do contexto da RG, as ondas gravitacionais representam uma configuração de curvatura em propagação. Em teorias de torção como o TERG [29], desenvolvidas no espaço de Weitzenböck, as ondas gravitacionais podem ser interpretadas como configurações de torção em propagação. Esta última interpretação possibilitou a descrição das ondas gravitacionais como defeitos topológicos no espaço-tempo [34, em analogia aos defeitos presentes em cristais.

Localmente, os elementos de linha 59 e 60 são equivalentes. Entretanto, globalmente existem certas escolhas que levam a efeitos globais distintos, como o caso do elemento de linha

$$
\begin{aligned}
d s^{2} & =H(u, \rho, \phi) d u^{2}+d \rho^{2}+\rho^{2} d \phi^{2} \\
& -2 J(u, \rho, \phi) d u d \phi+2 d u d v,
\end{aligned}
$$

onde $x=\rho \cos \phi$ e $y=\rho \sin \phi$. As ondas gravitacionais representadas pelo elemento de linha (67) são chamadas de ondas giratônicas [33, 35 37]. O tensor métrico que gera o elemento de linha (67) é, localmente, equivalente ao que gera o elemento de linha (60). Entretanto, como o efeito gravitacional é um efeito global, conforme visto na seção 2.3, não se pode argumentar que os campos gravitacionais sejam equivalentes. Pesquisas sobre as ondas giratônicas dentro do TERG levaram a obtenção de propriedades qualitativas distintas entre esses espaçostempo [38], corroborando a distinção entre os campos gravitacionais.

Destarte, o estudo das ondas gravitacionais planas fornece não apenas um campo de estudo introdutório para a RG, mas também um campo aberto de pesquisa para a investigação de resultados ainda não bem compreendidos na gravitação como um todo.

\section{Referências}

[1] A. Einstein, in: Das Relativitätsprinzip (Springer, Basel, 1923), p. 81.

[2] D. Hilbert, Mathematische Annalen 92, 1 (1924).

[3] A. Saa, Revista Brasileira de Ensino de Física 38, e4201 (2016).

[4] A.Z. Petrov, General Relativity and Gravitation 32, 1665 (2000).

[5] J.B. Griffiths e J. Podolskỳ, Exact space-times in Einstein's general relativity (Cambridge University Press, Cambridge, 2009).

[6] L.C.B. Crispino e M.C. Lima, Revista Brasileira de Ensino de Física 38, e4201 (2016).

[7] A. Einstein, Preuss. Akad. Wiss 1918, 154 (1918).

[8] A. Einstein e N. Rosen, Journal of the Franklin Institute 223, 43 (1937).

[9] H. Bondi, Nature 179, 1072 (1957).

[10] H. Bondi, F.A.E. Pirani e I. Robinson, Proceedings of the Royal Society of London 251, 519 (1959). 
[11] J. Weber, Physical Review Letters 22, 1320 (1969).

[12] J.M. Weisber, J.H. Taylor e L.A. Fowler, Scientific American 245, 74 (1981).

[13] S.M. Kopeikin, Physical Review D 56, 4455 (1997).

[14] O.D. Aguiar, L.A. Andrade, J.J. Barroso, P.J. Castro, C.A. Costa, S.T. Souza, A. Waard, A.C. Fauth, C. Frajuca, G. Frossati et al., Classical and Quantum Gravity 25, 114042 (2008).

[15] B.P. Abbott, R. Abbott, T.D. Abbott, M.R. Abernathy, F. Acernese, K. Ackley, C. Adams, T. Adams, P. Addesso, R.X. Adhikari et al., Physical Review Letters 116 , $061102(2016)$

[16] M. Cattani e J.M.F. Bassalo, Revista Brasileira de Ensino de Física 38, e4202 (2016).

[17] P.M. Zhang, C. Duval, G.W. Gibbons e P.A. Horvathy, Physics Letters B 772, 743 (2017).

[18] P.M. Zhang, C. Duval, G. W. Gibbons e P.A. Horvathy, Journal of Cosmology and Astroparticle Physics 2018, 030 (2018).

[19] P.M. Zhang, C. Duval, G.W. Gibbons e P.A. Horvathy, Physical Review D 96, 064013 (2017).

[20] J.W. Maluf, J.F. Rocha-Neto, S.C. Ulhoa e F.L. Carneiro, Gravitation and Cosmology 24, 261 (2018).

[21] M.P. Hobson, G.P. Efstathiou e A.N. Lasenby, General relativity: an introduction for physicists (Cambridge University Press, Cambridge, 2006).

[22] H. Stephani, Relativity: An introduction to special and general relativity (Cambridge University Press, Cambridge, 2004).

[23] M.P. Ramos E R.V. Maluf, Revista Brasileira de Ensino de Física 40, e2302 (2018).

[24] L.D. Landau E E.M. Lifshitz, The classical theory of fields (Elsevier, Amsterdam, 2013), v. 2.

[25] P.S. Letelier, Revista Brasileira de Ensino de Física 27, 121 (2005).

[26] I. Waga, Revista Brasileira de Ensino de Física 27, 57 (2005).

[27] R.G.G. Amorim, S.C. Ulhoa, P.M.M. Rocha e R.A.S. Paiva, Revista Brasileira de Ensino de Física 37, 2302 (2015).

[28] E. Poisson, A relativist's toolkit: the mathematics of black-hole mechanics (Cambridge University Press, Cambridge, 2004).

[29] J.W. Maluf, Annalen der Physik 525, 339 (2013).

[30] J.W. Maluf, General Relativity and Gravitation 46, 1734 (2014).

[31] H. Stephani, D. Kramer, M. MacCallum, C. Hoenselaers E E. Herlt, Exact solutions of Einstein's field equations (Cambridge University Press, Cambridge, 2009).

[32] H.W. Brinkmann, Mathematische Annalen 94, 119 (1925).

[33] J. Podolskỳ, R. Steinbauer e R. Švarc, Physical Review D 90, 044050 (2014).

[34] F.L. Carneiro, S.C. Ulhoa, J.W. Maluf e J.F. RochaNeto, The European Physical Journal C 81, 1 (2021).

[35] J.W. Maluf, J.F. Rocha-Neto, S.C. Ulhoa e F.L. Carneiro, Classical and Quantum Gravity 35, 115001 (2018).
[36] J. Podolskỳ, R. Švarc, R. Steinbauer e C. Sämann, Physical Review D 96, 064043 (2017).

[37] C. Sämann, R. Steinbauer e R. Švarc, Classical and Quantum Gravity 33, 215006 (2016).

[38] F.L. Carneiro, S.C. Ulhoa e J.F. Rocha-Neto, Physical Review D 100, 024023 (2019). 